DCPT-10/29, KUNS-2284

\title{
Stability of Holographic Superconductors
}

\author{
Sugumi Kanno ${ }^{1)}$ and Jiro Soda ${ }^{2}$ \\ 1) Centre for Particle Theory, Department of Mathematical Sciences, Durham University, \\ Science Laboratories, South Road, Durham, DH1 3LE, United Kingdom and \\ 2) Department of Physics, Kyoto University, Kyoto, 606-8501, Japan
}

(Dated: May 29, 2018)

\begin{abstract}
We study the dynamical stability of holographic superconductors. We first classify perturbations around black hole background solutions into vector and scalar sectors by means of a 2-dimensional rotational symmetry. We prove the stability of the vector sector by explicitly constructing the positive definite Hamiltonian. To reveal a mechanism for the stabilization of a superconducting phase, we construct a quadratic action for the scalar sector. From the action, we see the stability of black holes near a critical point is determined by the equation of motion for a charged scalar field. We show the effective mass of the charged scalar field in hairy black holes is always above the Breitenlohner-Freedman bound near the critical point due to the backreaction of a gauge field. It implies the stability of the superconducting phase. We also argue that the stability continues away from the critical point.
\end{abstract}

PACS numbers: 11.25.Tq,04.70.Bw,74.20.-z

\section{INTRODUCTION}

It is believed that the AdS/CFT correspondence is useful to study strongly coupled systems [1]. Remarkably, the AdS/CFT correspondence has been extended to the correspondence between gravity and condensed matter physics [2]. In particular, it has been shown that there exists a gravity dual of a superconductor [3 5]. Interestingly, the mean field square root behavior of the Landau-Ginzburg second order phase transition has been reproduced through the gravity dual of the superconductor. Moreover, it turned out that conductivity has similar features to that of the superconductor (see review articles and references therein [6, 7]).

It is well known that the superconductor can be explained as the second order phase transition phenomena. In terms of the Landau-Ginzburg theory, below a critical temperature, the effective mass of the order parameter field becomes tachyonic and destabilize normal phase. Eventually, a superconducting phase is realized as the new phase where the stability is guaranteed by a quartic potential. In the holographic description of the superconductor, the onset of the instability is well understood. The point is that the coupling of a charged scalar field to a gauge field through covariant derivatives induces an effective mass term for the scalar field [8]. This term becomes relevant as temperature gets lowered at fixed charge density, and eventually it makes the effective mass of the charged scalar field below the Breitenlohner-Freedman (BF) bound [9], and hence destabilize the system. On the other hand, the fate of the instability is not fully understood. In fact, in the gravity dual of superconductors, no quartic potential to stabilize the system after the instability is present. Hence, although a trigger of the phase transition is clear, a mechanism of stabilization of the superconducting phase is not apparent. In this paper, we study the dynamical stability of the superconducting phase in the gravity dual model of the superconductor and reveal that the superconductor is stabilized through a backreaction of the gauge field.

From the gravity point of view, the normal phase corresponds to charged black holes in anti-de Sitter spacetime, i.e., the Reissner-Nortström-AdS black holes. The instability below the critical temperature drives the black hole into hairy black hole which corresponds to the superconducting phase. Hence, what we would like to prove is the stability of this hairy black hole. Therefore, we can use the standard technique in the black hole perturbation theory [10 12]. We classify general perturbations around the hairy black hole into vector and scalar sectors by means of a 2-dimensional rotational symmetry of the black hole. We show the vector sector contains no unstable mode. In order to complete the proof of stability, we examine the scalar sector. By looking at the vicinity of the transition point, we demonstrate how the stability is realized in the superconducting phase. We also argue that the stability of the system persists away from the critical point.

The organization of this paper is as follows. In section 2, we introduce the model and present background equations of motion. Here, we describe the phase transition. In section 3, we explain the Arnowitt-Deser-Misner (ADM) formalism which is useful to perform efficient calculations and the stability analysis. In section 4 , we prove the stability of the vector sector by constructing the positive definite Hamiltonian. In section 5, we reveal a mechanism for the stabilization of the superconducting phase through the analysis of the scalar sector. Although the actual analysis is performed in the vicinity of the critical point, we argue the stability holds even away from the critical point. The final section is devoted to the conclusion. 


\section{GRAVITY DUAL OF SUPERCONDUCTORS}

In this section, we review the gravity/superconductor correspondence [3, 5]. Here, we take into account backreaction for completeness.

The action is given by

$$
S=\int d^{4} x \sqrt{-g}\left[\frac{1}{2 \kappa^{2}}\left(\mathcal{R}+\frac{6}{L^{2}}\right)-\frac{1}{4} F^{\mu \nu} F_{\mu \nu}-|\nabla \psi-i q A \psi|^{2}-V(\psi)\right],
$$

where $\mathcal{R}$ is the 4-dimensional Ricci scalar and $L$ denotes the AdS curvature scale. Here, we have incorporated the charged scalar field $\psi$ and the gauge field $A_{\mu}$ from which we can calculate the field strength $F_{\mu \nu}=\partial_{\mu} A_{\nu}-\partial_{\nu} A_{\mu}$. As for the potential $V(\psi)$, we take the mass term, $V(\psi)=m^{2}|\psi|^{2}$. In this paper, we assume that the mass is always above the BF bound. Note that the coupling constant $q$ controls the strength of the backreaction.

Let us consider the static background. Then, the metric is given by:

$$
d s_{b}^{2}=-f(r) e^{-\chi(r)} d t^{2}+\frac{d r^{2}}{f(r)}+\frac{r^{2}}{L^{2}} \delta_{a b} d y^{a} d y^{b}
$$

where $a, b$ denote $x, y$ coordinates. The other fields are expressed by:

$$
A_{\mu}=(\phi(r), 0,0,0), \quad \psi=\psi(r)
$$

Note that the scalar field is taken to be real using the $U(1)$ gauge transformation. Then, $t t$ and $r r$ components of the background Einstein equations yield

$$
\begin{aligned}
& f^{\prime}+\frac{1}{r} f-\frac{3 r}{L^{2}}+\kappa^{2} r\left[\frac{e^{\chi}}{2} \phi^{\prime 2}+m^{2} \psi^{2}+f\left(\psi^{\prime 2}+\frac{q^{2} \phi^{2} \psi^{2} e^{\chi}}{f^{2}}\right)\right]=0, \\
& \chi^{\prime}+2 \kappa^{2} r\left(\psi^{\prime 2}+\frac{q^{2} \phi^{2} \psi^{2} e^{\chi}}{f^{2}}\right)=0
\end{aligned}
$$

Here, a prime denotes derivative with respect to $r$. while the gauge and the scalar equations become

$$
\begin{aligned}
& \phi^{\prime \prime}+\left(\frac{\chi^{\prime}}{2}+\frac{2}{r}\right) \phi^{\prime}-\frac{2 q^{2} \psi^{2}}{f} \phi=0 \\
& \psi^{\prime \prime}+\left(\frac{f^{\prime}}{f}-\frac{\chi^{\prime}}{2}+\frac{2}{r}\right) \psi^{\prime}+\left(\frac{q^{2} \phi^{2} e^{\chi}}{f^{2}}-\frac{m^{2}}{f}\right) \psi=0 .
\end{aligned}
$$

From the last equation, we see the gauge potential $\phi$ induces the effective negative mass squared for the charged scalar field. This acts as a trigger of the phase transition.

By solving the above equations with appropriate boundary conditions, we obtain the asymptotic behavior

$$
\begin{aligned}
& \phi=\mu-\frac{\rho}{r}+\cdots, \\
& \psi=\frac{\left\langle\mathcal{O}_{\Delta}\right\rangle}{r^{\Delta}}+\cdots,
\end{aligned}
$$

where $\mu$ and $\rho$ are interpreted as a chemical potential and charge density of the dual theory on the boundary. $\left\langle\mathcal{O}_{\Delta}\right\rangle$ represents the expectation value of the operator $O_{\Delta}$ dual to the charged scalar field $\psi$. The exponent $\Delta$ is determined by the mass as $\Delta=3 / 2+\sqrt{9+4 m^{2} L^{2}} / 2$. This is the dictionary of AdS/CFT correspondence. When Hawking temperature is above a critical temperature, $T>T_{c}$, the solution is given by the Reissner-Nortström-AdS black holes

$$
\chi=\psi=0, \quad f=\frac{r^{2}}{L^{2}}-\frac{1}{r}\left(\frac{r_{+}^{3}}{L^{2}}+\frac{\kappa^{2} \rho^{2}}{2 r_{+}}\right)+\frac{\kappa^{2} \rho^{2}}{2 r^{2}}, \quad \phi=\rho\left(\frac{1}{r_{+}}-\frac{1}{r}\right)
$$

where $r_{+}$represents the horizon radius. For $T<T_{c}$, the Reissner-Nordström AdS black hole solutions become unstable and new hairy black holes will be firmed. There, the expectation value $\left\langle\mathcal{O}_{\Delta}\right\rangle$ has a non-trivial value proportional to $\sqrt{T_{c}-T}$. This is the phase transition from a normal phase to a superconducting phase.

In subsequent sections, we will investigate the stability of the hairy black holes corresponding to the superconducting phase. 


\section{USES OF ADM FORMALISM}

Although there are some indirect evidences of the stability of holographic superconductors [13, 14], it is important to give a direct evidence. To this end, we need to perform perturbative analysis of the system introduced in the previous section. In this section, we explain a useful method to obtain a quadratic action for black hole perturbations [15].

Since the background spacetime is static, it is useful to work in the ADM formalism. Let us take the parameterization for the metric

$$
d s^{2}=-N^{2} d t^{2}+h_{i j}\left(d x^{i}+N^{i} d t\right)\left(d x^{j}+N^{j} d t\right)
$$

where $i=r, x, y$ denotes the spatial coordinates, $N$ is the lapse function, and $N^{i}$ is the shift vector. Under this metric parameterization, the Einstein-Hilbert action can be written in terms of the spatial curvature $R$ and the extrinsic curvature $K_{i j}$ as

$$
\begin{aligned}
S_{\mathrm{R}} & =\frac{1}{2 \kappa^{2}} \int d^{4} x \sqrt{-g}\left[\mathcal{R}+\frac{6}{L^{2}}\right] \\
& =\frac{1}{2 \kappa^{2}} \int d t d^{3} x \sqrt{h}\left[N R+\frac{1}{N}\left(E^{i j} E_{i j}-E^{2}\right)+N \frac{6}{L^{2}}\right]
\end{aligned}
$$

where for convenience we used the symmetric tensor $E_{i j}$ instead of the extrinsic curvature, which is defined by

$$
E_{i j}=\frac{1}{2}\left[\dot{h}_{i j}-N_{i ; j}-N_{j ; i}\right]=N K_{i j}
$$

Here, a dot and a semicolon are a time derivative and a covariant derivative with respect to $h_{i j}$, respectively. Similarly, the action for the gauge field reads

$$
\begin{aligned}
S_{\mathrm{A}} & =\int d^{4} x \sqrt{-g}\left[-\frac{1}{4} F^{\mu \nu} F_{\mu \nu}\right] \\
& =\int d^{4} x \sqrt{h} N\left[\frac{1}{2 N^{2}} h^{i j} F_{t i} F_{t j}-\frac{N^{i}}{N^{2}}\left(h^{j \ell}-\frac{N^{j} N^{\ell}}{N^{2}}\right) F_{t j} F_{i \ell}-\frac{1}{4}\left(h^{i k}-\frac{N^{i} N^{k}}{N^{2}}\right)\left(h^{j \ell}-\frac{N^{j} N^{\ell}}{N^{2}}\right) F_{i j} F_{k \ell}\right] .
\end{aligned}
$$

The action for the charged scalar field is also written as

$$
\begin{aligned}
S_{\psi}=\int d^{4} x \sqrt{-g}[ & \left.-|\nabla \psi-i q A \psi|^{2}-V(\psi)\right] \\
=\int d^{4} x \sqrt{h} N[ & \frac{1}{N^{2}}\left\{\dot{\psi}^{*}+i q A_{t} \psi^{*}-N^{i}\left(\psi_{, i}^{*}+i q A_{i} \psi^{*}\right)\right\}\left\{\dot{\psi}-i q A_{t} \psi-N^{j}\left(\psi, j-i q A_{j} \psi\right)\right\} \\
& \left.-h^{i j}\left(\psi_{, i}^{*}+i q A_{i} \psi^{*}\right)\left(\psi_{, j}-i q A_{j} \psi\right)-V(\psi)\right] .
\end{aligned}
$$

Now, it is easy to see why the ADM formalism is useful for our purpose. In the ADM formalism, the background metric reads

$$
d s_{b}^{2}=-N^{2}(r) d t^{2}+h_{i j}(r) d x^{i} d x^{j}
$$

where

$$
N^{2}(r)=f(r) e^{-\chi(r)}, \quad h_{i j}(r) d x^{i} d x^{j}=\frac{d r^{2}}{f(r)}+\frac{r^{2}}{L^{2}} \delta_{a b} d y^{a} d y^{b}
$$

Apparently, there is no shift vector $N^{i}$ in the background metric. Hence, we find that $E_{i j}$ vanishes for the background. And, the gauge field has only time component, so we have $F_{i j}=0$. Thus, some of the terms in Eqs. (11), (13) and (14) are already the second order quantities. In this way, the ADM formalism makes calculations for obtaining the quadratic action easier. In order to further simplify the calculations, we take the variation of the total action $S=S_{R}+S_{A}+S_{\psi}$ with respect to the lapse function $N$ to yield the Hamiltonian constraint equation:

$$
\frac{1}{2 \kappa^{2}}\left(R+\frac{6}{L^{2}}\right)-\frac{1}{2 N^{2}} h^{i j} F_{t i} F_{t j}-\frac{1}{N^{2}} q^{2} A_{t}^{2} \psi^{*} \psi-h^{i j} \psi_{, i}^{*} \psi, j-V(\psi)=0 .
$$


Substituting this equation into the total action, we can simplify the part proportional to $(\sqrt{h} N)^{(2)}$ as

$$
\int d^{4} x(\sqrt{h} N)^{(2)}\left[\frac{1}{N^{2}} h^{i j} F_{t i} F_{t j}+\frac{2}{N^{2}} q^{2} A_{t}^{2} \psi^{*} \psi\right],
$$

where index (2) means the second order quantity.

Since there exists a 2-dimensional plane symmetry in the black hole background, we can decompose any tensor into vector and scalar sectors by means of the 2-dimensional rotation symmetry. Those sectors are decoupled in the linear equations. Hence, we will consider the vector and the scalar sectors separately.

\section{VECTOR SECTOR}

In this section, we will prove the stability of the vector sector of perturbations. To this aim, we use the formalism explained in the previous section. Hereafter, we set $L=1$ and $\kappa^{2}=1$.

Let us consider the metric perturbations on the background metric (15),

$$
d s^{2}=d s_{b}^{2}+\delta g_{\mu \nu} d x^{\mu} d x^{\nu} .
$$

The vector sector of metric perturbations is generally expressed by

$$
\delta g_{\mu \nu}=\left(\begin{array}{ccc}
0 & 0 & \delta g_{t a} \\
* & 0 & \delta g_{r a} \\
* & * & \delta g_{a b}
\end{array}\right), \quad * \text { is symmetric part }
$$

where $\delta g_{t a}, \delta g_{r a}$ and $\delta g_{a b}=2 \zeta_{(a \mid b)}$ satisfy the divergence free condition: $\delta g_{t a} \mid a=\delta g_{r a}{ }^{\mid a}=\zeta_{a}{ }^{\mid a}=0$. Here, $\mid$ represents a partial derivative. Using the gauge transformation $x^{\mu} \rightarrow x^{\mu}-\xi^{\mu}$ with

$$
\xi_{\mu}=\left(0,0, \xi_{a}\right)
$$

where $\xi_{a}{ }^{\mid a}=0$, those variables transform into

$$
\delta g_{t a} \rightarrow \delta g_{t a}+\dot{\xi}_{a}, \quad \delta g_{r a} \rightarrow \delta g_{r a}+\xi_{a}^{\prime}-\frac{2}{r} \xi_{a}, \quad \delta g_{a b} \rightarrow \delta g_{a b}+2 \xi_{(a \mid b)}
$$

Thus we can make $\delta g_{a b}$ vanish by choosing $\xi_{a}=-\zeta_{a}$ and we get the complete gauge fixing. This is called the Regge-Wheeler gauge. In the Regge-Wheeler gauge, the perturbations that belong to the vector sector can be written as

$$
\delta g_{\mu \nu}=\left(\begin{array}{ccc}
0 & 0 & v_{a} \\
* & 0 & w_{a} \\
* & * & 0
\end{array}\right)
$$

where $v_{a}^{\mid a}=w_{a}^{\mid a}=0$. In the ADM formalism, this corresponds to

$$
\delta N=0, \quad \delta N_{i}=v_{i}, \quad \delta h_{i j}=\left(\begin{array}{cc}
0 & w_{a} \\
* & 0
\end{array}\right) .
$$

As to the gauge field, we can take

$$
\delta A_{\mu}=\left(0,0, Z_{a}\right)
$$

where $Z_{a}$ satisfies $Z_{a}^{\mid a}=0$.

Now, we can calculate the quadratic action from Eqs. (11), (13) and (14). The quadratic part of Eq. (11) is going to be

$$
\begin{aligned}
S_{R}= & \frac{1}{2} \int d^{4} x(\sqrt{h} N)^{(0)}\left[R+\frac{1}{N^{2}}\left(E^{i j} E_{i j}-E^{2}\right)\right]^{(2)} \\
= & \frac{1}{2} \int d^{4} x r^{2} e^{-\frac{\chi}{2}}\left[-\frac{f^{2} \chi^{\prime}}{r^{3}} w^{a} w_{a}+\left.\frac{f}{2 r^{4}} w^{a} w_{a}\right|_{\mid b}+\frac{1}{2 r^{2}} e^{\chi}\left(\dot{w}_{a}-v_{a}^{\prime}+\frac{2}{r} v_{a}\right)\left(\dot{w}^{a}-v^{\prime a}+\frac{2}{r} v^{a}\right)\right. \\
& \left.-\frac{1}{2 r^{4} f} e^{\chi} v^{a} v_{a}^{\mid b} \mid b\right]
\end{aligned}
$$


The quadratic part of Eq. (13) becomes

$$
\begin{aligned}
S_{\mathrm{A}}= & \int d^{4} x(\sqrt{h} N)^{(0)}\left[\frac{1}{2 N^{2}} h^{i j} F_{t i} F_{t j}-\frac{N^{i}}{N^{2}} h^{j \ell} F_{t j} F_{i \ell}-\frac{1}{4} h^{i k} h^{j \ell} F_{i j} F_{k \ell}\right]^{(2)} \\
& +\int d^{4} x(\sqrt{h} N)^{(2)}\left[\frac{1}{N^{2}} h^{i j} F_{t i} F_{t j}\right]^{(0)} \\
= & \int d^{4} x\left[e^{\frac{\chi}{2}} \phi^{\prime} w^{a} \dot{Z}_{a}+\frac{1}{2 f} e^{\frac{\chi}{2}} \dot{Z}_{a} \dot{Z}^{a}-e^{\frac{\chi}{2}} \phi^{\prime} v^{a} Z_{a}^{\prime}-\frac{f}{2} e^{-\frac{\chi}{2}} Z^{\prime a} Z_{a}^{\prime}-\frac{1}{2 r^{2}} e^{-\frac{\chi}{2}} Z_{a \mid c} Z^{a \mid c}\right] .
\end{aligned}
$$

The quadratic part of Eq. (14) gives

$$
\begin{aligned}
S_{\psi}= & \int d^{4} x(\sqrt{h} N)^{(0)}\left[\frac{1}{N^{2}}\left\{\dot{\psi}^{*}+i q A_{t} \psi^{*}-N^{i}\left(\psi_{, i}^{*}+i q A_{i} \psi^{*}\right)\right\}\left\{\dot{\psi}-i q A_{t} \psi-N^{j}\left(\psi_{, j}-i q A_{j} \psi\right)\right\}\right. \\
& \left.-h^{i j}\left(\psi_{, i}^{*}+i q A_{i} \psi^{*}\right)\left(\psi_{, j}-i q A_{j} \psi\right)-V(\psi)\right]^{(2)} \\
& +\int d^{4} x(\sqrt{h} N)^{(2)}\left[\frac{2}{N^{2}} q^{2} A_{t}^{2} \psi^{*} \psi\right]^{(0)} \\
= & \int d^{4} x\left[-2 \frac{q^{2}}{f} e^{\frac{\chi}{2}} \phi \psi^{2} v^{a} Z_{a}-f^{2} e^{-\frac{\chi}{2}} \psi^{\prime 2} w^{a} w_{a}-q^{2} e^{-\frac{\chi}{2}} \psi^{2} Z^{a} Z_{a}-q^{2} e^{\frac{\chi}{2}} \phi^{2} \psi^{2} w^{a} w_{a}\right] .
\end{aligned}
$$

The total quadratic action for the vector sector becomes

$$
\begin{aligned}
S= & S_{\mathrm{R}}+S_{\mathrm{A}}+S_{\psi} \\
= & \frac{1}{2} \int d t d r d^{2} k e^{-\frac{\chi}{2}}\left[-\frac{\chi^{\prime}}{r} f^{2} w^{a} w_{a}-\frac{k_{b}^{2} f}{2 r^{2}} w^{a} w_{a}+\frac{e^{\chi}}{2}\left(\dot{w}_{a}-v_{a}^{\prime}+\frac{2}{r} v_{a}\right)\left(\dot{w}^{a}-v^{\prime a}+\frac{2}{r} v^{a}\right)+\frac{k_{b}^{2}}{2 r^{2} f} e^{\chi} v^{a} v_{a}\right] \\
& +\int d t d r d^{2} k\left[e^{\frac{\chi}{2}} \phi^{\prime} w^{a} \dot{Z}_{a}+\frac{1}{2 f} e^{\frac{\chi}{2}} \dot{Z}_{a} \dot{Z}^{a}-e^{\frac{\chi}{2}} \phi^{\prime} v^{a} Z_{a}^{\prime}-\frac{f}{2} e^{-\frac{\chi}{2}} Z^{\prime a} Z_{a}^{\prime}-\frac{k_{b}^{2}}{2 r^{2}} e^{-\frac{\chi}{2}} Z_{a} Z^{a}\right. \\
& \left.-2 \frac{q^{2}}{f} e^{\frac{\chi}{2}} \phi \psi^{2} v^{a} Z_{a}-f^{2} e^{-\frac{\chi}{2}} \psi^{\prime 2} w^{a} w_{a}-q^{2} e^{-\frac{\chi}{2}} \psi^{2} Z^{a} Z_{a}-q^{2} e^{\frac{\chi}{2}} \phi^{2} \psi^{2} w^{a} w_{a}\right]
\end{aligned}
$$

where we moved on to the Fourier space with respect to $x, y$ coordinates. Note that there are three unknown variable $v_{a}, w_{a}$ and $Z_{a}$, which satisfy the transverse conditions. Among them, $v_{a}$ is not a dynamical one which should be eliminated.

The action Eq. (28) becomes,

$$
\begin{aligned}
S=\int d t d r d^{2} k e^{-\frac{\chi}{2}}[ & -\frac{k_{b}^{2} f}{4 r^{2}} w^{a} w_{a}+\frac{e^{\chi}}{4}\left(\dot{w}_{a}-v_{a}^{\prime}+\frac{2}{r} v_{a}\right)\left(\dot{w}^{a}-v^{a}+\frac{2}{r} v^{a}\right)+\frac{k_{b}^{2}}{4 r^{2} f} e^{\chi} v^{a} v_{a}+e^{\chi} \phi^{\prime} w^{a} \dot{Z}_{a} \\
& \left.+\frac{1}{2 f} e^{\chi} \dot{Z}_{a} \dot{Z}^{a}-e^{\chi} \phi^{\prime} v^{a} Z_{a}^{\prime}-\frac{f}{2} Z^{\prime a} Z_{a}^{\prime}-\frac{k_{b}^{2}}{2 r^{2}} Z_{a} Z^{a}-2 \frac{q^{2}}{f} e^{\chi} \phi \psi^{2} v^{a} Z_{a}-q^{2} \psi^{2} Z^{a} Z_{a}\right],
\end{aligned}
$$

where we eliminated some terms proportional to $w_{a} w^{a}$ using the background equation (4). Now we want to eliminate the non-dynamical field $v^{a}$ by completing the square with respect to $v_{a}$. In doing so, we need the Hamiltonian formalism in order to eliminate $v_{a}^{\prime} v^{\prime a}$. Defining the conjugate momentum

$$
\begin{aligned}
& P_{w}^{a}=\frac{\partial \mathcal{L}}{\partial \dot{w}_{a}}=\frac{1}{2} e^{\frac{\chi}{2}}\left(\dot{w}^{a}-v^{a}+\frac{2}{r} v^{a}\right), \\
& P_{z}^{a}=\frac{\partial \mathcal{L}}{\partial \dot{Z}_{a}}=e^{\frac{\chi}{2}}\left(\phi^{\prime} w^{a}+\frac{1}{f} \dot{Z}^{a}\right),
\end{aligned}
$$

we obtain the Hamiltonian

$$
\begin{aligned}
H & =\int d r d^{2} k_{a}\left[P_{w}^{a} \dot{w}_{a}+P_{z}^{a} \dot{Z}_{a}-\mathcal{L}\right] \\
& =\int d r d^{2} k_{a}\left[e^{-\frac{\chi}{2}} P_{w}^{a} P_{w a}+\left(v_{a}^{\prime}-\frac{2}{r} v_{a}\right) P_{w}^{a}+\frac{f}{2} e^{-\frac{\chi}{2}}\left(P_{z}^{a}-e^{\frac{\chi}{2}} \phi^{\prime} w^{a}\right)\left(P_{z a}-e^{\frac{\chi}{2}} \phi^{\prime} w_{a}\right)\right.
\end{aligned}
$$




$$
\begin{aligned}
& +\frac{k_{b}^{2} f}{4 r^{2}} e^{-\frac{\chi}{2}} w^{a} w_{a}-\frac{k_{b}^{2}}{4 r^{2} f} e^{\frac{\chi}{2}} v^{a} v_{a}+e^{\frac{\chi}{2}} \phi^{\prime} v^{a} Z_{a}^{\prime}+\frac{f}{2} e^{-\frac{\chi}{2}} Z_{a}^{\prime} Z^{\prime a} \\
& \left.+\frac{k_{c}^{2}}{2 r^{2}} e^{-\frac{\chi}{2}} Z_{a} Z^{a}+2 \frac{q^{2}}{f} e^{\frac{\chi}{2}} \phi \psi^{2} v^{a} Z_{a}+q^{2} e^{-\frac{\chi}{2}} \psi^{2} Z^{a} Z_{a}\right] .
\end{aligned}
$$

Let us see the terms containing $v_{a}$. After integrating by parts for $v_{a}^{\prime}$, we have

$$
-v_{a}\left(P_{w}^{a \prime}+\frac{2}{r} P_{w}^{a}-e^{\frac{\chi}{2}} \phi^{\prime} Z_{a}^{\prime}-2 \frac{q^{2}}{f} e^{\frac{\chi}{2}} \phi \psi^{2} Z^{a}\right)-\frac{k_{b}^{2}}{4 r^{2} f} e^{\frac{\chi}{2}} v^{a} v_{a}
$$

By completing the square for the second and third terms as

$$
\begin{gathered}
-\frac{1}{4 r^{2} f} e^{\frac{\chi}{2}} k_{b}^{2}\left\{v^{a}+\frac{2 r^{2} f}{k_{b}^{2}} e^{-\frac{\chi}{2}}\left(P_{w}^{a \prime}+\frac{2}{r} P_{w}^{a}-e^{\frac{\chi}{2}} \phi^{\prime} Z_{a}^{\prime}-2 \frac{q^{2}}{f} e^{\frac{\chi}{2}} \phi \psi^{2} Z^{a}\right)\right\}^{2} \\
+\frac{r^{2} f}{k_{b}^{2}} e^{-\frac{\chi}{2}}\left\{P_{w}^{a \prime}+\frac{2}{r} P_{w}^{a}-e^{\frac{\chi}{2}} \phi^{\prime} Z_{a}^{\prime}-2 \frac{q^{2}}{f} e^{\frac{\chi}{2}} \phi \psi^{2} Z^{a}\right\}^{2} .
\end{gathered}
$$

the variable $v_{a}$ can be eliminated because the first squared term vanishes after substituting the equation of motion for $v_{a}$. Then the Hamiltonian becomes

$$
\begin{aligned}
H=\int d r d^{2} k[ & \frac{r^{2} f}{k_{b}^{2}} e^{-\frac{\chi}{2}}\left\{P_{w}^{a \prime}+\frac{2}{r} P_{w}^{a}-e^{\frac{\chi}{2}} \phi^{\prime} Z_{a}^{\prime}-2 \frac{q^{2}}{f} e^{\frac{\chi}{2}} \phi \psi^{2} Z^{a}\right\}^{2} \\
& +e^{-\frac{\chi}{2}} P_{w}^{a} P_{w a}+\frac{f}{2} e^{-\frac{\chi}{2}}\left(P_{z}^{a}-e^{\frac{\chi}{2}} \phi^{\prime} w^{a}\right)\left(P_{z a}-e^{\frac{\chi}{2}} \phi^{\prime} w_{a}\right)+\frac{k_{b}^{2} f}{4 r^{2}} e^{-\frac{\chi}{2}} w^{a} w_{a} \\
& \left.+\frac{f}{2} e^{-\frac{\chi}{2}} Z_{a}^{\prime} Z^{\prime a}+\frac{k_{c}^{2}}{2 r^{2}} e^{-\frac{\chi}{2}} Z_{a} Z^{a}+q^{2} e^{-\frac{\chi}{2}} \psi^{2} Z^{a} Z_{a}\right] .
\end{aligned}
$$

We find the Hamiltonian consists of positive terms. This implies the stability of the system. The argument is as follows. Suppose an unstable mode exists, then the Hamiltonian must grow. However, since the Lagrangian has a time translation invariance, the energy of the system is conserved. This contradicts the growth of the Hamiltonian. Therefore, the unstable mode cannot exist in the vector sector. Note that this is true either for the normal phase or the superconducting phase.

\section{SCALAR SECTOR}

In the previous section, we have proved the stability of the vector sector for both the normal and superconducting phase. Thus, if unstable modes exist, they must belong to the scalar sector. Above the critical temperature, the system is the Reissner-Nordström-AdS black hole with a trivial scalar field. For this case, we know the system is stable [16]. Below the critical temperature, the effective mass of the charged scalar field violates BF bound, then the system ceases to be stable. Eventually, a hairy black hole is formed. It is believed that the new hairy black hole is stable. In this section, we reveal the stabilization mechanism of the hairy black hole.

The scalar sector of metric perturbations is generally expressed by seven degrees of freedom

$$
\delta g_{\mu \nu}=\left(\begin{array}{ccc}
\delta g_{t t} & \delta g_{t r} & \delta g_{t a} \\
* & \delta g_{r r} & \delta g_{r a} \\
* & * & \delta g_{a b}
\end{array}\right), \quad * \text { is symmetric part }
$$

where $\delta g_{a b}=\zeta_{1} \delta_{a b}+\zeta_{2 \mid a b}$ has two degrees of freedom in the scalar sector. Using the gauge transformation with

$$
\xi_{\mu}=\left(\xi_{t}, \xi_{r}, \xi_{\mid a}\right)
$$

metric perturbations transform as

$$
\delta g_{t t} \rightarrow \delta g_{t t}+2 \dot{\xi}_{t}-f^{2} e^{-\chi}\left(\frac{f^{\prime}}{f}-\chi^{\prime}\right) \xi_{r}
$$




$$
\begin{aligned}
\delta g_{t r} & \rightarrow \delta g_{t r}+\xi_{t}^{\prime}+\dot{\xi}_{r}-\left(\frac{f^{\prime}}{f}-\chi^{\prime}\right) \xi_{t}, \\
\delta g_{t a} & \rightarrow \delta g_{t a}+\xi_{t \mid a}+\dot{\xi}_{\mid a} \\
\delta g_{r r} & \rightarrow \delta g_{r r}+2 \xi_{r}^{\prime}+\frac{f^{\prime}}{f} \xi_{r} \\
\delta g_{r a} & \rightarrow \delta g_{r a}+\xi_{r \mid a}+\xi_{\mid a}^{\prime}-\frac{2}{r} \xi_{\mid a} \\
\delta g_{a b} & \rightarrow \delta g_{a b}+2 \xi_{\mid a b}+2 f r \xi_{r} \delta_{a b}
\end{aligned}
$$

Using the gauge degree of freedom of $\xi_{t}$, we can eliminate $\delta g_{t a}$. Using the gauge degree of freedom of $\xi$ and $\xi_{r}$, we can take $\delta g_{a b}=0$. Therefore, we take the following gauge.

$$
\delta g_{\mu \nu}=\left(\begin{array}{ccc}
N^{2} \bar{H} & H_{1} & 0 \\
* & H / f & w_{\mid a} \\
* & * & 0
\end{array}\right) . \quad * \text { is symmetric part. }
$$

where the components of metric perturbations $w, H, H_{1}$ and $\bar{H}$ depend on $(t, r, a)$. In the ADM formalism, we can put

$$
\delta N=N(\sqrt{1-\bar{H}}-1), \quad \delta N_{r}=H_{1}, \quad \delta h_{i j}=\left(\begin{array}{cc}
H / f & w_{\mid a} \\
* & 0
\end{array}\right) .
$$

In addition to the metric perturbations, we need to consider fluctuations of the gauge field $\delta A_{\mu}$ and the charged scalar field $\delta \psi, \delta \psi^{*}$. As to the gauge field, we can take

$$
\delta A_{\mu}=\left(\lambda, \alpha, \beta_{\mid a}\right) .
$$

Using the $U(1)$ gauge transformation, we can take the scalar field perturbations to be real $\delta \psi^{*}=\delta \psi$. However, for the trivial background $\psi=0$, this gauge fixing is singular because the phase loses its meaning when the amplitude is zero.

Now, we can calculate the quadratic action for perturbed quantities using the ADM formalism. The action for the gravity is calculated as

$$
\begin{gathered}
S_{\mathrm{R}}=\frac{1}{2} \int d^{4} x\left[(\sqrt{h} N)^{(0)} R^{(2)}+(\sqrt{h} N)^{(1)} R^{(1)}+\left(\frac{\sqrt{h}}{N}\right)^{(0)}\left(E^{i j} E_{i j}-E^{2}\right)^{(2)}\right] \\
=\frac{1}{2} \int d^{4} x e^{-\frac{\chi}{2}}\left[-\frac{1}{2} r^{2} \bar{H}\left(2 \frac{f}{r} H^{\prime}-\frac{1}{r^{2}} H^{\mid a}{ }_{\mid a}+2 \frac{f}{r^{2}} H+2 \frac{f^{\prime}}{r} H+2 \frac{f}{r^{2}} w_{\mid a}^{\prime a}+\frac{f^{\prime}}{r^{2}} w^{\mid a}{ }_{\mid a}+2 \frac{f}{r^{3}} w^{\mid a}{ }_{\mid a}\right)\right. \\
+\frac{r f}{2}\left(\frac{1}{r}+\frac{f^{\prime}}{f}-\frac{3}{2} \chi^{\prime}\right) H^{2}-\frac{f}{2}\left(\frac{f^{\prime}}{f}+\frac{2}{r}-\chi^{\prime}\right) H^{\mid a} w_{\mid a}-\frac{f^{2} \chi^{\prime}}{r} w^{\mid a} w_{\mid a} \\
\left.+\frac{e^{\chi}}{2}\left(\dot{w}-H_{1}\right)_{\mid a}\left(\dot{w}-H_{1}\right)^{\mid a}+2 r e^{\chi} H_{1} \dot{H}+r f \chi^{\prime} e^{\chi} H_{1}^{2}\right] .
\end{gathered}
$$

In vacuum case, $\chi$ vanishes and the above action gives the Zerilli equation [11]. In the present case, we have other fields. The action for the gauge field becomes

$$
\begin{aligned}
S_{A}=\int d^{4} x[ & (\sqrt{h} N)^{(0)}\left\{\frac{1}{2 N^{2}} h^{i j} F_{t i} F_{t j}-\frac{N^{i}}{N^{2}} h^{j \ell} F_{t j} F_{i \ell}-\frac{1}{4} h^{i k} h^{j \ell} F_{i j} F_{k \ell}\right\}^{(2)} \\
+ & \left.(\sqrt{h} N)^{(1)}\left\{\frac{1}{2 N^{2}} h^{i j} F_{t i} F_{t j}\right\}^{(1)}+(\sqrt{h} N)^{(2)}\left\{\frac{1}{N^{2}} h^{i j} F_{t i} F_{t j}\right\}^{(0)}\right] \\
=\int d^{4} x r^{2} e^{\frac{\chi}{2}}[ & \frac{1}{2}\left\{\dot{\alpha}-\lambda^{\prime}+\frac{1}{2} \phi^{\prime}(H-\bar{H})\right\}^{2}+\frac{1}{r^{2}} \phi^{\prime} w^{\mid a}(\dot{\beta}-\lambda)_{\mid a}+r^{2} \phi^{\prime} \gamma^{\mid a}\left(\alpha-\beta^{\prime}\right)_{\mid a} \\
& \left.+\frac{1}{2 r^{2} f}(\dot{\beta}-\lambda)_{\mid a}(\dot{\beta}-\lambda)^{\mid a}-\frac{f}{2 r^{2}} e^{-\chi}\left(\beta^{\prime}-\alpha\right)_{\mid a}\left(\beta^{\prime}-\alpha\right)^{\mid a}\right] .
\end{aligned}
$$


Since we have eliminated the phase of the charged scalar field, the corresponding physical degree is absorbed by the gauge field. Then, $\alpha$ and $\beta$ are dynamical degrees describing the massive gauge field. The time component of the perturbed vector $\lambda$ is a Lagrange multiplier and hence not dynamical. Finally, the action for the scalar field is given by

$$
\begin{aligned}
S_{\psi}= & \int d^{4} x(\sqrt{h} N)^{(0)}\left[\frac{1}{N^{2}}\left\{\dot{\psi}+i q A_{t} \psi-N^{i}\left(\psi_{, i}+i q A_{i} \psi\right)\right\}\left\{\dot{\psi}-i q A_{t} \psi-N^{j}\left(\psi_{, j}-i q A_{j} \psi\right)\right\}\right. \\
& \left.-h^{i j}\left(\psi_{, i}+i q A_{i} \psi\right)\left(\psi_{, j}-i q A_{j} \psi\right)-V(\psi)\right]^{(2)} \\
+ & \quad d^{4} x(\sqrt{h} N)^{(1)}\left[\frac{1}{N^{2}}\left\{\dot{\psi}+i q A_{t} \psi-N^{i}\left(\psi_{, i}+i q A_{i} \psi\right)\right\}\left\{\dot{\psi}-i q A_{t} \psi-N^{j}\left(\psi_{, j}-i q A_{j} \psi\right)\right\}\right. \\
& +\int d^{4} x(\sqrt{h} N)^{(2)}\left[\frac{2}{N^{2}} q^{2} A_{t}^{2} \psi^{2}\right]^{(0)} \\
= & \int d^{4} x r^{2} e^{-\frac{\chi}{2}}\left[\frac{e^{\chi}}{f}\left(\delta \dot{\psi}^{2}-2 f \psi^{\prime} H_{1} \delta \dot{\psi}+f^{2} \psi^{\prime 2} H_{1}^{2}\right)-2 q^{2} e^{\chi} \phi \psi^{2} H_{1} \alpha+q^{2} \phi^{2} \delta \psi^{2}+q^{2} \psi^{2} \lambda^{2}\right. \\
& +4 q^{2} \phi \psi \delta \psi \lambda-q^{2} f \psi^{2} \alpha^{2}-\frac{q^{2}}{r^{2}} \psi^{2} \beta^{\mid a} \beta_{\mid a}-f \delta \psi^{\prime 2}-\frac{1}{r^{2}} \delta \psi_{\mid a} \delta \psi^{\mid a} \\
& \left.-f \psi^{\prime 2} H^{2}-\frac{f^{2}}{r^{2}} \psi^{\prime 2} w^{\mid a} w_{\mid a}+2 f \psi^{\prime} H \delta \psi^{\prime}+2 \frac{f}{r^{2}} \psi^{\prime} w^{\mid a} \delta \psi_{\mid a}-m^{2} \delta \psi^{2}\right] \\
& +\int d^{4} x \frac{1}{2} r^{2} e^{-\frac{\chi}{2}}(H-\bar{H})\left[2 q^{2} \phi \psi^{2} \lambda+2 q^{2} \phi^{2} \psi \delta \psi+f \psi^{\prime 2} H-2 f \psi^{\prime} \delta \psi^{\prime}-2 m^{2} \psi \delta \psi\right] \\
& +\int d^{4} x 2 \frac{q^{2} r^{2}}{f} e^{\frac{\chi}{2}} \phi^{2} \psi^{2}\left[-\frac{1}{8} \bar{H}^{2}-\frac{1}{8} H^{2}-\frac{f}{2 r^{2}} w^{\mid a} w_{\mid a}-\frac{1}{4} \bar{H} H\right]
\end{aligned}
$$

Note that we have eight unknown variables $\bar{H}, H_{1}, H, w, \lambda, \alpha, \beta, \delta \psi$. Among these, only four variables are physical. Thus, the total action in Fourier space becomes

$$
\begin{aligned}
S=\frac{1}{2} \int d t d r d^{2} k[- & \frac{1}{2} r^{2} e^{-\frac{\chi}{2}} \bar{H}\left(2 \frac{f}{r} H^{\prime}+\frac{k_{a}^{2}}{r^{2}} H+2 \frac{f}{r^{2}} H+2 \frac{f^{\prime}}{r} H-2 \frac{k_{a}^{2} f}{r^{2}} w^{\prime}-\frac{k_{a}^{2} f^{\prime}}{r^{2}} w-2 \frac{k_{a}^{2} f}{r^{3}} w\right) \\
& +\frac{r f}{2}\left(\frac{1}{r}+\frac{f^{\prime}}{f}-\frac{3}{2} \chi^{\prime}\right) e^{-\frac{\chi}{2}} H^{2}-\frac{k_{a}^{2} f}{2}\left(\frac{f^{\prime}}{f}+\frac{2}{r}-\chi^{\prime}\right) e^{-\frac{\chi}{2}} H w \\
& \left.+\frac{k_{a}^{2}}{2} e^{\frac{\chi}{2}}\left(\dot{w}-H_{1}\right)^{2}+2 r e^{\frac{\chi}{2}} H_{1} \dot{H}+r f \chi^{\prime} e^{\frac{\chi}{2}} H_{1}^{2}\right] \\
+ & \int d t d r d^{2} k r^{2} e^{\frac{\chi}{2}}\left[\frac{1}{2}\left\{\dot{\alpha}-\lambda^{\prime}+\frac{1}{2} \phi^{\prime}(H-\bar{H})\right\}^{2}+\frac{k_{a}^{2}}{r^{2}} \phi^{\prime} w(\dot{\beta}-\lambda)+\frac{k_{a}^{2}}{2 r^{2} f}(\dot{\beta}-\lambda)^{2}-\frac{k_{a}^{2} f}{2 r^{2}} e^{-\chi}\left(\beta^{\prime}-\alpha\right)^{2}\right] \\
+ & \int d t d r d^{2} k r^{2} e^{-\frac{\chi}{2}}\left[\frac{e^{\chi}}{f}\left(\delta \dot{\psi}-f \psi^{\prime} H_{1}\right)^{2}-2 q^{2} e^{\chi} \phi \psi^{2} H_{1} \alpha+q^{2} \phi^{2} \delta \psi^{2}+q^{2} \psi^{2} \lambda^{2}+4 q^{2} \phi \psi \delta \psi \lambda-q^{2} f \psi^{2} \alpha^{2}\right. \\
& \left.-\frac{k_{a}^{2} q^{2}}{r^{2}} \psi^{2} \beta^{2}-f \delta \psi^{\prime 2}-\frac{k_{a}^{2}}{r^{2}} \delta \psi^{2}-f \psi^{\prime 2} H^{2}+2 f \psi^{\prime} \delta \psi^{\prime} H+2 \frac{k_{a}^{2} f}{r^{2}} \psi^{\prime} w \delta \psi-m^{2} \delta \psi^{2}\right] \\
+ & \int d t d r d^{2} k \frac{1}{2} r^{2} e^{-\frac{\chi}{2}}(H-\bar{H})\left[2 q^{2} \phi \psi^{2} \lambda+2 q^{2} \phi^{2} \psi \delta \psi+f \psi^{\prime 2} H-2 f \psi^{\prime} \delta \psi^{\prime}-2 m^{2} \psi \delta \psi\right] \\
+ & \int d t d r d^{2} k r^{2} e^{\frac{\chi}{2}} \frac{2 q^{2}}{f} \phi^{2} \psi^{2}\left[-\frac{1}{8} \bar{H}^{2}-\frac{1}{8} H^{2}-\frac{1}{4} \bar{H} H\right]
\end{aligned}
$$

where Eq. (4) is used.

Now, it is convenient to use the Hamiltonian formalism for getting an insight. Defining the canonical conjugate momentum:

$$
\begin{aligned}
& P_{w}=\frac{\partial \mathcal{L}}{\partial \dot{w}}=\frac{k_{a}^{2}}{2} e^{\frac{\chi}{2}}\left(\dot{w}-H_{1}\right), \\
& P_{\alpha}=\frac{\partial \mathcal{L}}{\partial \dot{\alpha}}=r^{2} e^{\frac{\chi}{2}}\left\{\dot{\alpha}-\lambda^{\prime}+\frac{1}{2} \phi^{\prime}(H-\bar{H})\right\},
\end{aligned}
$$




$$
\begin{aligned}
P_{\beta} & =\frac{\partial \mathcal{L}}{\partial \dot{\beta}}=k_{a}^{2} e^{\frac{\chi}{2}} \phi^{\prime} w+\frac{k_{a}^{2}}{f} e^{\frac{\chi}{2}}(\dot{\beta}-\lambda), \\
P_{\delta \psi} & =\frac{\partial \mathcal{L}}{\partial \dot{\delta} \psi}=2 \frac{r^{2}}{f} e^{\frac{\chi}{2}}\left(\delta \dot{\psi}-f \psi^{\prime} H_{1}\right),
\end{aligned}
$$

we can perform the Legendre transformation and obtain the Hamiltonian density

$$
\begin{aligned}
\mathcal{H}= & \frac{1}{k_{a}^{2}} e^{-\frac{\chi}{2}} P_{w}^{2}+\frac{1}{2 r^{2}} e^{-\frac{\chi}{2}} P_{\alpha}^{2}+\frac{f}{2 k_{a}^{2}} e^{-\frac{\chi}{2}} P_{\beta}^{2}+\frac{f}{4 r^{2}} e^{-\frac{\chi}{2}} P_{\delta \psi}^{2} \\
& +P_{w} H_{1}+f \psi^{\prime} P_{\delta \psi} H_{1}-\frac{1}{2} \phi^{\prime} P_{\alpha}(H-\bar{H})-f \phi^{\prime} P_{\beta} w+P_{\alpha} \lambda^{\prime}+P_{\beta} \lambda \\
& +\frac{r f}{2} e^{-\frac{\chi}{2}} \bar{H} H^{\prime}+\frac{r f}{4} e^{-\frac{\chi}{2}}\left(\frac{k_{a}^{2}}{r f}+\frac{2}{r}+2 \frac{f^{\prime}}{f}\right) \bar{H} H-\frac{k_{a}^{2} f}{2} e^{-\frac{\chi}{2}} \bar{H} w^{\prime}-\frac{k_{a}^{2} f}{4} e^{-\frac{\chi}{2}}\left(\frac{f^{\prime}}{f}+\frac{2}{r}\right) \bar{H} w \\
& -\frac{r f}{4}\left(\frac{1}{r}+\frac{f^{\prime}}{f}-\frac{3}{2} \chi^{\prime}\right) e^{-\frac{\chi}{2}} H^{2}+r^{2} f e^{-\frac{\chi}{2}} \psi^{\prime 2} H^{2}-\frac{r^{2} f}{2} e^{-\frac{\chi}{2}} \psi^{\prime 2} H(H-\bar{H})+\frac{q^{2} r^{2}}{4 f} e^{\frac{\chi}{2}} \phi^{2} \psi^{2}(H+\bar{H})^{2} \\
& +\frac{k_{a}^{2} f}{4}\left(\frac{f^{\prime}}{f}+\frac{2}{r}-\chi^{\prime}\right) e^{-\frac{\chi}{2}} H w-r e^{-\frac{\chi}{2}} H_{1} \dot{H}-\frac{r f}{2} \chi^{\prime} e^{\frac{\chi}{2}} H_{1}^{2}+\frac{k_{a}^{2} f}{2} e^{\frac{\chi}{2}} \phi^{\prime 2} w^{2} \\
& +\frac{k_{a}^{2} f}{2} e^{-\frac{\chi}{2}}\left(\beta^{\prime}-\alpha\right)^{2}+2 q^{2} r^{2} e^{\frac{\chi}{2}} \phi \psi^{2} H_{1} \alpha+q^{2} r^{2} f e^{-\frac{\chi}{2}} \psi^{2} \alpha^{2}+k_{a}^{2} q^{2} e^{-\frac{\chi}{2}} \psi^{2} \beta^{2} \\
& -2 r^{2} f e^{-\frac{\chi}{2}} \psi^{\prime} H \delta \psi^{\prime}-2 k_{a}^{2} f e^{-\frac{\chi}{2}} \psi^{\prime} w \delta \psi-\frac{r^{2}}{2} e^{-\frac{\chi}{2}}(H-\bar{H})\left(2 q^{2} \phi^{2} \psi \delta \psi-2 f \psi^{\prime} \delta \psi^{\prime}-2 m^{2} \psi \delta \psi\right) \\
& +r^{2} f e^{-\frac{\chi}{2}} \delta \psi^{\prime 2}+k_{a}^{2} e^{-\frac{\chi}{2}} \delta \psi^{2}+r^{2} e^{-\frac{\chi}{2}} m^{2} \delta \psi^{2} \\
& -q^{2} r^{2} e^{-\frac{\chi}{2}} \phi^{2} \delta \psi^{2}-q^{2} r^{2} e^{-\frac{\chi}{2}} \psi^{2}\left\{\lambda^{2}+4 \frac{\phi}{\psi} \delta \psi \lambda+\phi(H-\bar{H}) \lambda\right\}
\end{aligned}
$$

First, we consider the normal phase $\psi=0$. In this case, we find the scalar field perturbation decouples from the other parts in Eq. (50) and reads

$$
\mathcal{H}_{\delta \psi}=\frac{f}{4 r^{2}} e^{-\frac{\chi}{2}} P_{\delta \psi}^{2}+r^{2} f e^{-\frac{\chi}{2}} \delta \psi^{\prime 2}+k_{a}^{2} e^{-\frac{\chi}{2}} \delta \psi^{2}+r^{2} e^{-\frac{\chi}{2}} m^{2} \delta \psi^{2}-q^{2} r^{2} e^{-\frac{\chi}{2}} \phi^{2} \delta \psi^{2} .
$$

As the Reissner-Nordström-AdS black hole is known to be stable, the instability of the system should be caused by the scalar field. Apparently, the last term in Eq. (51) could destabilize the system. Indeed, as we lower the Hawking temperature, the potential $\phi \propto 1 / r$ becomes larger near the horizon. Then, the effective mass $m_{\text {eff }}^{2}=m^{2}-q^{2} \phi^{2}$ gets smaller. Eventually, below the critical temperature, the effective mass $m_{\text {eff }}^{2}=m^{2}-q^{2} \phi^{2}$ violates the BF bound and the system becomes unstable.

Now, we see what happens in the superconducting phase $\psi \neq 0$. In order to make the discussion clear, we focus on the stability of the region near the critical point where $\psi$ is close to zero and the Reissner-Nordström-AdS black hole becomes a good approximation. The scalar perturbation $\delta \psi$ decouples from the metric perturbation in the ReissnerNordström-AdS limit $\psi \rightarrow 0$. Hence, in the limit, we can set $\bar{H}=H_{1}=H=w=0$ when we look at the scalar field. In a sense, this is close to the probe limit. Note that we have four variables $\alpha, \beta, \lambda, \delta \psi$ among which only $\lambda$ is unphysical. Now, the relevant part of the Hamiltonian is given by

$$
\begin{aligned}
H=\int d r d^{2} k_{a}[ & \frac{1}{2 r^{2}} e^{-\frac{\chi}{2}} P_{\alpha}^{2}+\frac{f}{2 k_{a}^{2}} e^{-\frac{\chi}{2}} P_{\beta}^{2}+\frac{f}{4 r^{2}} e^{-\frac{\chi}{2}} P_{\delta \psi}^{2}+\frac{k_{a}^{2} f}{2} e^{-\frac{\chi}{2}}\left(\beta^{\prime}-\alpha\right)^{2}+q^{2} r^{2} f e^{-\frac{\chi}{2}} \psi^{2} \alpha^{2}+k_{a}^{2} q^{2} e^{-\frac{\chi}{2}} \psi^{2} \beta^{2} \\
& +r^{2} f e^{-\frac{\chi}{2}} \delta \psi^{\prime 2}+k_{a}^{2} e^{-\frac{\chi}{2}} \delta \psi^{2}+r^{2} e^{-\frac{\chi}{2}} m^{2} \delta \psi^{2} \\
& \left.-q^{2} r^{2} e^{-\frac{\chi}{2}} \phi^{2} \delta \psi^{2}-q^{2} r^{2} e^{-\frac{\chi}{2}} \psi^{2}\left(\lambda^{2}+4 \frac{\phi}{\psi} \delta \psi \lambda+\frac{P_{\alpha}^{\prime}-P_{\beta}}{q^{2} r^{2} e^{-\frac{\chi}{2}} \psi^{2}} \lambda\right)\right]
\end{aligned}
$$

At first sight, the system seems to be unstable because of the term, $-q^{2} r^{2} e^{-\frac{\chi}{2}} \phi^{2} \delta \psi^{2}$ in the last line of Eq. (52) in the limit of the Reissner-Nordström AdS $\psi \rightarrow 0$. However, $\psi$ is not actually zero. We can eliminate the unphysical variable $\lambda$ by completing the square for $\lambda$ or alternatively by using the equation of motion for $\lambda$

$$
\lambda=-2 \frac{\phi}{\psi} \delta \psi-\frac{P_{\alpha}^{\prime}-P_{\beta}}{2 q^{2} r^{2} e^{-\frac{x}{2}} \psi^{2}} .
$$


Then, the last line of Eq. (52) becomes

$$
+3 q^{2} r^{2} e^{-\frac{\chi}{2}} \phi^{2} \delta \psi^{2}+2 \frac{\phi}{\psi}\left(P_{\alpha}^{\prime}-P_{\beta}\right) \delta \psi+\frac{\left(P_{\alpha}^{\prime}-P_{\beta}\right)^{2}}{4 q^{2} r^{2} e^{-\frac{\chi}{2}} \psi^{2}} .
$$

The effect we have incorporated is merely the backreaction of the gauge field. Notice that the constraint equation, which is derived by the variation with respect to $\lambda$,

$$
P_{\alpha}^{\prime}-P_{\beta}=0
$$

holds in the limit $\psi \rightarrow 0$. This constraint equation is nothing but the Gauss law for the gauge field. This appears because the limit $\psi \rightarrow 0$ recovers the gauge invariance in the gauge field system. Therefore, in the vicinity of the critical point, we can ignore last two terms in Eq. (54). Thus, when we take the limit $\psi \rightarrow 0$ from the side of the superconducting phase, the Hamiltonian reduces to

$$
\begin{aligned}
H=\int d r d^{2} k_{a}[ & \frac{1}{2 r^{2}} e^{-\frac{\chi}{2}} P_{\alpha}^{2}+\frac{f}{2 k_{a}^{2}} e^{-\frac{\chi}{2}} P_{\beta}^{2}+\frac{f}{4 r^{2}} e^{-\frac{\chi}{2}} P_{\delta \psi}^{2}+\frac{k_{a}^{2} f}{2} e^{-\frac{\chi}{2}}\left(\beta^{\prime}-\alpha\right)^{2} \\
& \left.+r^{2} f e^{-\frac{\chi}{2}} \delta \psi^{\prime 2}+k_{a}^{2} e^{-\frac{\chi}{2}} \delta \psi^{2}+r^{2} e^{-\frac{\chi}{2}} m^{2} \delta \psi^{2}+3 q^{2} r^{2} e^{-\frac{\chi}{2}} \phi^{2} \delta \psi^{2}\right] .
\end{aligned}
$$

Now, it is clear that the Hamiltonian is positive definite except for the mass term. Moreover, the effective mass squared $m_{\text {eff }}^{2}=m^{2}+3 q^{2} \phi^{2}$ is always above the BF bound. Thus, we find the system is going to be stable after the phase transition. It should be stressed that the flip of the sign of the term proportional to $\phi^{2} \delta \psi^{2}$ is possible only in the condensed phase with $\psi \neq 0$ everywhere. In fact, there is an (infinite) set of solutions for $\psi$ which are regular on the horizon and satisfy the required boundary condition at infinity. Based on our analysis, we believe that the solutions with nodes are likely to be unstable and only the lowest solution with no nodes is stable.

We can argue the stability of the superconducting phase far from the critical point as follows. From the point of view of the gravity dual, the phase transition is the bifurcation of the solutions at the phase transition point from where two branches are developed. The normal branch is apparently unstable. What we have shown is the stability of the other branch in the vicinity of the bifurcation point. However, from the continuity, this is sufficient to prove the stability of the superconducting phase provided that there exists no other bifurcation point on this branch. Indeed, it is difficult to imagine a new bifurcation point because the sign flip of the term proportional to $\phi^{2} \delta \psi^{2}$ persists as long as the scalar field has the expectation value. Admittedly, this argument does not give a proof of stability far away from the critical point.

\section{CONCLUSION}

We have studied the dynamical stability of superconductors using the ADM formalism. First, we proved the stability of the vector sector by explicitly constructing the positive definite conserved Hamiltonian. We have also obtained the Hamiltonian for the scalar sector. In the normal phase, the action decoupled into two parts. One is that of the Reissner-Nordström-AdS black hole and the other is that of the charged scalar field in the Reissner-Nordström-AdS background. It is well known that the former system is stable. The stability of the latter system depends on the effective mass of the scalar field. Since the effective mass becomes more negative as the gauge potential becomes large, the phase transition occurs at the critical temperature. And, below the critical temperature, the ReissnerNordström-AdS black holes are unstable. Numerically, we know the scalar hair is developed below $T_{c}$. For this case, the structure of the constraint for $\lambda$ changes. After eliminating the unphysical variable $\lambda$, we found the effective mass became above the BF bound. In particular, near the critical temperature $\psi \rightarrow 0$, we have decoupled equations for the charged scalar field, which is now stable. Thus, we have revealed a mechanism for the stabilization of superconducting phase in the scalar sector. The result is remarkable because the stability near the critical point is guaranteed by the non-gravitational effect. In fact, the effect of the gauge field changes the signature of the effective additional mass when the charged scalar field has condensation. In our argument, we have assumed the solution for the scalar field has no node. Therefore, other solutions with nodes are expected to be unstable. Admittedly, we have not been able to prove the stability of superconductors far away from the critical point. However, the fact that the effective mass squared remains to be above the BF bound in the superconducting phase suggests the stability of holographic superconductors. It should be stressed that the stabilization mechanism we found originates from the structure of the minimal gauge coupling, hence the result applies to holographic superconductors in any dimensions.

Our analysis can be extended to various holographic superconductor models. For example, it is possible to extend our analysis to the Gauss-Bonnet superconductors [17 21]. In that case, again, we can use the results in black hole perturbations [22, 23]. 
More importantly, we need to study the dynamical stability of holographic superconductors embedded into string theory [24 26]. However, it requires a more sophisticated method for the analysis.

\section{Acknowledgments}

SK would like to thank TAP members in Kyoto University for warm hospitality. Most of this work was done while SK was visiting Kyoto University supported by JSPS Grant-in-Aid for Scientific Research on Innovative Area No.21111006. SK is supported by an STFC rolling grant. JS is supported by the Grant-in-Aid for Scientific Research Fund of the Ministry of Education, Science and Culture of Japan No.22540274, the Grant-in-Aid for Scientific Research (A) (No. 22244030), the Grant-in-Aid for Scientific Research on Innovative Area No.21111006 and the Grant-in-Aid for the Global COE Program "The Next Generation of Physics, Spun from Universality and Emergence".

[1] J. M. Maldacena, Adv. Theor. Math. Phys. 2, 231 (1998) [Int. J. Theor. Phys. 38, 1113 (1999)] arXiv:hep-th/9711200.

[2] S. A. Hartnoll, arXiv:0903.3246 [hep-th].

[3] S. A. Hartnoll, C. P. Herzog and G. T. Horowitz, Phys. Rev. Lett. 101, 031601 (2008) arXiv:0803.3295 [hep-th]].

[4] G. T. Horowitz and M. M. Roberts, Phys. Rev. D 78, 126008 (2008) arXiv:0810.1077] [hep-th]].

[5] S. A. Hartnoll, C. P. Herzog and G. T. Horowitz, JHEP 0812, 015 (2008) arXiv:0810.1563 [hep-th]].

[6] C. P. Herzog, arXiv:0904.1975 [hep-th].

[7] G. T. Horowitz, arXiv:1002.1722 [hep-th].

[8] S. S. Gubser, arXiv:0801.2977 [hep-th].

[9] P. Breitenlohner and D. Z. Freedman, Annals Phys. 144, 249 (1982).

[10] T. Regge and J. A. Wheeler, Phys. Rev. 108, 1063 (1957).

[11] F. J. Zerilli, Phys. Rev. Lett. 24, 737 (1970).

[12] V. Moncrief, Annals Phys. 88, 323 (1974).

[13] K. Maeda, S. Fujii and J. i. Koga, Phys. Rev. D 81, 124020 (2010) arXiv:1003.2689 [gr-qc]].

[14] K. Murata, S. Kinoshita and N. Tanahashi, arXiv:1005.0633 [hep-th].

[15] T. Takahashi and J. Soda, arXiv:1005.0286 [gr-qc].

[16] S.Chandrasekar, The Mathematical Theory of Black Holes, Oxford University Press, 1983.

[17] R. Gregory, S. Kanno and J. Soda, JHEP 0910, 010 (2009) arXiv:0907.3203 [hep-th]].

[18] Q. Pan, B. Wang, E. Papantonopoulos, J. Oliveira and A. B. Pavan, Phys. Rev. D 81, 106007 (2010) arXiv:0912.2475 [hep-th]].

[19] Y. Brihaye and B. Hartmann, Phys. Rev. D 81, 126008 (2010) arXiv:1003.5130 [hep-th]].

[20] Y. Liu, Q. Pan, B. Wang and R. G. Cai, arXiv:1007.2536 [hep-th].

[21] R. G. Cai, Z. Y. Nie and H. Q. Zhang, arXiv:1007.3321 [hep-th].

[22] G. Dotti and R. J. Gleiser, Class. Quant. Grav. 22, L1 (2005) arXiv:gr-qc/0409005;

G. Dotti and R. J. Gleiser, Phys. Rev. D 72, 044018 (2005) arXiv:gr-qc/0503117.

[23] T. Takahashi and J. Soda, Phys. Rev. D 79, 104025 (2009) arXiv:0902.2921 [gr-qc]];

T. Takahashi and J. Soda, Phys. Rev. D 80, 104021 (2009) arXiv:0907.0556 [gr-qc]];

T. Takahashi and J. Soda, in preparation.

[24] S. S. Gubser, C. P. Herzog, S. S. Pufu and T. Tesileanu, Phys. Rev. Lett. 103, 141601 (2009) arXiv:0907.3510 [hep-th]].

[25] J. P. Gauntlett, J. Sonner and T. Wiseman, Phys. Rev. Lett. 103, 151601 (2009) arXiv:0907.3796 [hep-th]].

[26] J. P. Gauntlett, J. Sonner and T. Wiseman, JHEP 1002, 060 (2010) arXiv:0912.0512 [hep-th]]. 